\title{
Oromucosal Gel Dosage Form
}

National Cancer Institute

\section{Source}

National Cancer Institute. Oromucosal Gel Dosage Form. NCI Thesaurus. Code C149735.

Semi-solid single-dose or multidose preparation consisting of a hydrophilic gel intended

for oromucosal use. It is applied to the oral cavity or onto a specific part of the oral cavity, to obtain a local effect. 